\title{
Dramatic Reduction of the Effect of Nanoconfinement on the Glass Transition of Polymer Films via Addition of Small-Molecule Diluent
}

\author{
Christopher J. Ellison, ${ }^{1}$ Robert L. Ruszkowski, ${ }^{1}$ Nathaniel J. Fredin, ${ }^{1}$ and John M. Torkelson ${ }^{1,2, *}$ \\ ${ }^{1}$ Department of Chemical and Biological Engineering, Northwestern University, Evanston, Illinois 60208, USA \\ ${ }^{2}$ Department of Materials Science and Engineering, Northwestern University, Evanston, Illinois 60208, USA
}

(Received 2 July 2003; published 5 March 2004; publisher error corrected 11 March 2004)

\begin{abstract}
The effect of nanoconfinement on the glass transition temperature $T_{g}$ in thin polymer films is studied as a function of added small-molecule diluent or plasticizer. The decrease [increase] in $T_{g}$ found in nanoconfined, neat polystyrene [poly(2-vinyl pyridine)] is suppressed by added diluent, with $13-20 \mathrm{~nm}$ thick polystyrene films exhibiting bulk $T_{g}$ upon addition of $9 \mathrm{wt} \%$ pyrene or $4 \mathrm{wt} \%$ dioctylphthalate. This is explained by a connection between the size scale of the cooperative dynamics associated with $T_{g}$, which decreases with added diluent, and the size scale of the nanoconfinement effect.
\end{abstract}

The deviation of the glass transition temperature, $T_{g}$, from its bulk value in nanoconfined polymers has been studied by ellipsometry [1,2], x-ray reflectivity [3], and other techniques [4-11], raising many fundamental questions [11,12]. Reviews $[2,11]$ have shown substantial agreement among the many results on the $T_{g}$-nanoconfinement effect for supported polystyrene (PS) films, where decreases in $T_{g}$ are observed with decreasing thickness below $\sim 60-80 \mathrm{~nm}$. With attractive polymer-substrate interactions, e.g., hydrogen bonding between poly(2-vinyl pyridine) (P2VP) and a silicon substrate with a native oxide surface layer, increases in $T_{g}$ may be observed with decreasing thickness [3,6], caused by a reduction of cooperative segmental mobility near the substrate. Simulations [13] have reiterated the role of substrate-polymer interactions in determining whether $T_{g}$ increases or decreases with decreasing thickness.

While many studies [1-13] have focused on the thickness dependence of $T_{g}$ for neat polymer films, such materials do not fully represent the realm of important nanoconfined polymer systems. For example, photoacid generators as additives in polymeric photoresists are a key enabling technology for the reduction to sub-100 $\mathrm{nm}$ feature sizes in microelectronic devices [14]. Furthermore, photoresist processing parameters such as acid diffusivity depend on the proximity of processing conditions to $T_{g}$ [15]. At a fundamental level, the presence of small-molecule diluents in polymers reduces the extent of cooperativity by relaxing constraints on cooperative segmental mobility defining $T_{g}$ [16,17]. As there has been interest in connecting the length scale associated with cooperative segmental mobility to that at which $T_{g}$-nanoconfinement effects are observed [11], the addition of diluents to polymer allows the length scale of cooperativity to be altered without otherwise changing polymer structure or polymer-substrate interactions, allowing for a critical, qualitative test of whether these two length scales are connected. This study, which uses fluorescence to characterize $T_{g}$ in polymer films [6-8], is the first investigation of the impact of small-molecule diluents on the $T_{g}$-nanoconfinement effect and demonstrates the dramatic suppression of this effect upon diluent addition.

Polystyrene [Pressure Chemical; $M_{n}=263 \mathrm{~kg} / \mathrm{mol}$, $M_{w} / M_{n}=1.10$ by gel permeation chromatography (GPC); $T_{g}$ onset $=373 \mathrm{~K}$ by differential scanning calorimetry (DSC), second heat at $10 \mathrm{~K} / \mathrm{min}$ ], P2VP (Polysciences; nominal $M_{n}=200 \mathrm{~kg} / \mathrm{mol}, M_{w} / M_{n}=1.7$; $T_{g}$ onset $=372 \mathrm{~K}$ by DSC), pyrene $(99+\%$, Aldrich) and dioctylphthalate (DOP) (99\%, Aldrich) were used as received. Pyrene-labeled PS $\left(M_{n}=440 \mathrm{~kg} / \mathrm{mol}\right.$, $M_{w} / M_{n}=1.73$ or $M_{n}=90 \mathrm{~kg} / \mathrm{mol}, M_{w} / M_{n}=1.78$ by GPC; both with $T_{g}$ onset $=369 \mathrm{~K}$ by DSC) was synthesized by initiating styrene polymerization with benzoyl peroxide at $348 \mathrm{~K}$ in the presence of a trace of 1-pyrenylbutyl methacrylate. This yielded a polymer with a 170 to 1 ratio of styrene- to pyrene-labeled methacrylate repeat units (UV absorbance). Pyrene-labeled PS was dissolved in toluene and precipitated in methanol at least 5 times to remove the residual monomer (confirmed by fluorescence-detection GPC) and placed in a vacuum oven at $378 \mathrm{~K}$ for $3 \mathrm{~d}$ prior to use. See Ref. [7] for synthesis of 1-pyrenylbutyl methacrylate.

Pyrene-doped PS (P2VP) films were spin coated onto glass from toluene (butanol). Pyrene-labeled PS films and DOP-doped, pyrene-labeled PS films were spin coated from toluene solutions onto $\mathrm{NaCl}$ IR crystal windows, transferred to glass in a water reservoir by floating, and dried in ambient conditions for several hours prior to taking measurements. Thickness was characterized by profilometry, using the average of at least ten measurements.

For pyrene-doped PS, fluorescence was measured using excitation at $322 \mathrm{~nm}$ and monitoring emission from $370-500 \mathrm{~nm}$; emission peaks from the pyrene monomer (single pyrene units) are at $\sim 374,385$, and $395 \mathrm{~nm}$, and the maximum emission from the excimer (two pyrene units forming excited-state dimer) is located 
at $\sim 480 \mathrm{~nm}$. (No excimer is present at $0.2 \mathrm{wt} \%$ pyrene.) For pyrene-labeled PS, fluorescence was measured using excitation at $340 \mathrm{~nm}$ and monitoring emission from 374-405 $\mathrm{nm}$; emission peaks are at $\sim 378$ and $398 \mathrm{~nm}$. Intensities were measured as a function of temperature upon cooling from above $T_{g}$. Details are reported in Refs. [6-8].

The temperature $(T)$ dependence of fluorescence of PS films is shown as a function of pyrene content in Fig. 1. The $T_{g}$ is identified by the intersection of the fits to linear regions in the $T$ dependences of the rubbery- and glassystate fluorescence. (This fluorescence method provides sensitivity to $T_{g}$ through changes in local density of the nanoscale environment surrounding the pyrene chromophore, with a slightly denser environment yielding a higher intensity [8]. Sensitivity to $T_{g}$ has also been reported using the intrinsic fluorescence of polystyrene, albeit for films several microns thick [7].) The 485- and 24-nm-thick PS films doped with $0.2 \mathrm{wt} \%$ pyrene have $T_{g} \mathrm{~s}$ of 373 and $362 \mathrm{~K}$, respectively, while the 183- and 13 -nm-thick PS films doped with $9 \mathrm{wt} \%$ pyrene have $T_{g} \mathrm{~s}$ of 352 and $353 \mathrm{~K}$ (error of $\pm 1 \mathrm{~K}$ ), respectively. A reduction in bulk $T_{g}$ with an increasing diluent is an expected plasticization effect. These results indicate that the reduction of $T_{g}$ in neat PS due to nanoconfinement is suppressed by a high concentration of pyrene diluent. Also noteworthy is that with the $0.2 \mathrm{wt} \%$ pyrene system the apparent strength in the transition at $T_{g}$ (difference between the rubbery- and the glassy-state $T$ dependences of fluorescence) is severely reduced in ultrathin films. Ellipsometry of neat PS films [2] has also shown a weakening in the strength of the transition at $T_{g}$ with decreasing thickness. In stark contrast, the 9 wt \% pyrene-doped PS films have rubbery- and glassy-state $T$ dependences that are nearly independent of thickness, indicating that thin and ultrathin films exhibit similar strengths of the transition at $T_{g}$, with little impact of nanoconfinement.

Figure 2 shows the thickness dependence of $T_{g}-T_{g}$ (bulk) for PS films as a function of pyrene content.
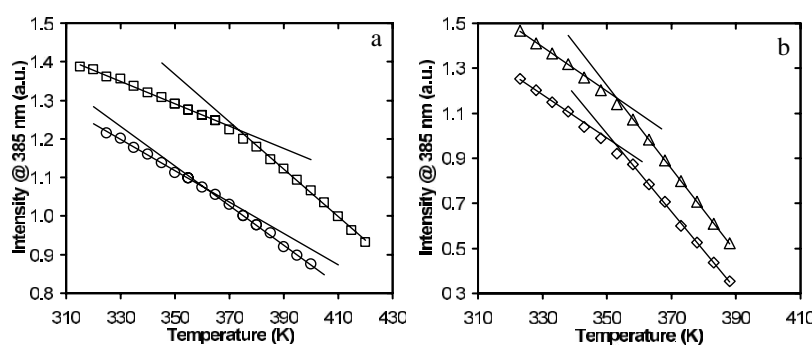

FIG. 1. Temperature dependence of the fluorescence intensity for PS films doped with pyrene: PS films doped with $0.2 \mathrm{wt} \%$ pyrene (a) are $485 \mathrm{~nm}(\square)$ and $24 \mathrm{~nm}(\bigcirc)$ thick; PS films doped with 9 wt $\%$ pyrene (b) are $183 \mathrm{~nm}(\triangle)$ and $13 \mathrm{~nm}(\diamond)$ thick. Intensities have been normalized to $373 \mathrm{~K}$ and arbitrarily shifted vertically for clarity.
The bulk $T_{g} \mathrm{~s}$ as measured by fluorescence for $0.2,2$, and $9 \mathrm{wt} \%$ pyrene-doped PS are 373,369 , and $351 \mathrm{~K}$, respectively, in reasonable agreement with bulk $T_{g} \mathrm{~s}$ obtained by DSC of 373,367 , and $356 \mathrm{~K}$, respectively. Remarkably, while $0.2 \mathrm{wt} \%$ pyrene-doped PS films yield a thickness-dependent $T_{g}$ in agreement with ellipsometry data on neat PS films $[1,2,6]$, the addition of 9 wt $\%$ pyrene to PS films results in a thickness-independent $T_{g}$ in films as thin as $13 \mathrm{~nm}$ where neat PS films exhibit a $T_{g}$ depression of $\sim 40 \mathrm{~K}$. The impact of confinement is "tunable" as shown by the $2 \mathrm{wt} \%$ pyrene-doped PS which exhibits a reduction in $T_{g}$ between those of 0.2 and $9 \mathrm{wt} \%$ pyrene-doped PS.

While pyrene reduces $T_{g}$ and the degree to which confinement alters $T_{g}$ properties, it is not a traditional diluent. In order to show generality of the suppression of nanoconfinement effects in PS films by the addition of small-molecule diluents, the plasticizer DOP was added to pyrene-labeled PS, yielding the results in Fig. 3. Here the diluent (DOP) is distinct from the fluorescence label (pyrene) that reports $T_{g}$. [Pyrene-doped $(0.2 \mathrm{wt} \%)$ and pyrene-labeled PS have identical $T_{g}$-nanoconfinement behavior in neat supported films [8].] The bulk $T_{g} \mathrm{~s}$ as measured by fluorescence for 0,2 , and $4 \mathrm{wt} \%$ DOP-doped pyrene-labeled PS films are 371,365 , and $362 \mathrm{~K}$, respectively, in reasonable agreement with bulk $T_{g}$ values obtained via DSC of 369,367 , and $360 \mathrm{~K}$, respectively. Figure 3 shows that the ability of DOP to suppress the nanoconfinement effect in PS films is similar to that observed when pyrene is the diluent. These results also demonstrate that a PS film containing a diluent can have a higher $T_{g}$ than a PS film of identical thickness lacking diluent, e.g., a 14-nm-thick PS film containing $4 \mathrm{wt} \%$ DOP has a $T_{g}$ that is $\sim 27 \mathrm{~K}$ higher than that of a neat PS film of identical thickness.

The latter result may have important technological implications. It suggests that if polymeric features of size less than $100 \mathrm{~nm}$ are required for a particular application,

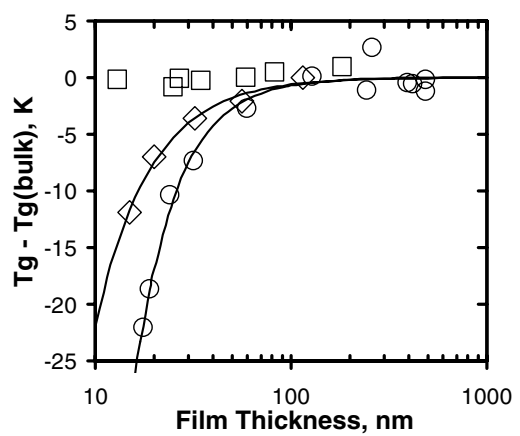

FIG. 2. $T_{g}-T_{g}$ (bulk) for PS films doped with $0.2 \mathrm{wt} \%(\bigcirc)$, $2 \mathrm{wt} \%(\diamond)$, and 9 wt $\%(\square)$ pyrene. The curves represent least squares fits of the data to the empirical relation $T_{g}(h)=$ $T_{g}$ (bulk) $\left[1-(A / h)^{\delta}\right]$ originally proposed by Keddie et al. [1] yielding parameter values $A=4.3 \mathrm{~nm}$ and $\delta=2.0$ for $0.2 \mathrm{wt} \%$ pyrene and $A=2.0 \mathrm{~nm}$ and $\delta=1.7$ for $2 \mathrm{wt} \%$ pyrene. 


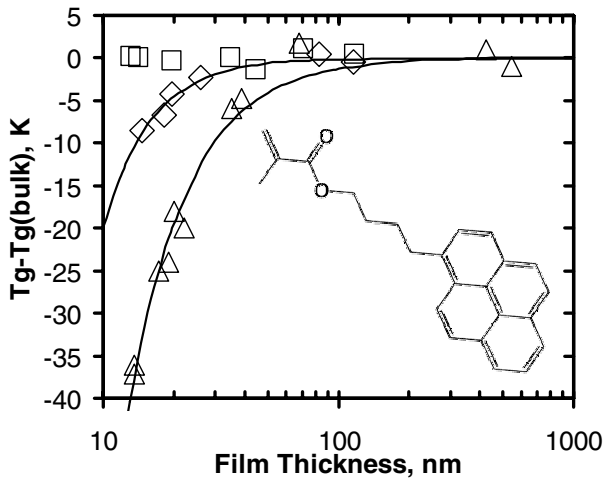

FIG. 3. $T_{g}-T_{g}$ (bulk) for neat $(\triangle)$, and 2 wt $\%(\diamond)$, or 4 wt $\%$ ( $\square$ ) DOP-doped pyrene-labeled PS films. The curves represent least squares fits of the data to the empirical relation originally proposed by Keddie et al. [1] yielding parameter values $A=$ $2.6 \mathrm{~nm}$ and $\delta=2.1$ for $2 \mathrm{wt} \%$ DOP-doped pyrene-labeled PS and $A=3.5 \mathrm{~nm}$ and $\delta=1.7$ for neat pyrene-labeled PS. The inset shows the structure of the 1-pyrenylbutyl methacrylate monomer used to label PS.

the presence of small-molecule diluents may be beneficial. If a reduction in bulk $T_{g}$ due to diluents is tolerable, the reward is that $T_{g}$-nanoconfinement effects may be reduced or avoided entirely.

The reduction in the nanoconfinement effect can be understood by considering how diluents affect cooperative dynamics. A fluorescence study of pyrene-labeled PS in single layers of bulk, multilayer PS films [8] has shown that free-surface layers (at the air-polymer interface) with thicknesses below $40-60 \mathrm{~nm}$ have $T_{g}$ s reduced relative to bulk and that ultrathin layers separated from the free surface by $10-30 \mathrm{~nm}$ also have reduced $T_{g} \mathrm{~s}$. It was argued that a perturbation in cooperative dynamics at the free surface can propagate at reduced strength into the film over $\sim 30 \mathrm{~nm}$ because the gradient in average cooperative segmental dynamics (the dynamics that define $T_{g}$ ) across a film cannot be too sharp. In other words, the average cooperative dynamics associated with $T_{g}$ at a particular film depth are impacted by the average cooperativity present in layers several tens of nanometers away from the layer of interest, a prerequisite for a smooth transition from enhanced dynamics at the free surface to bulk dynamics in the interior. Instead, the distance over which the gradient persists is comparable to the length scale needed to encompass the breadth of cooperative segmental dynamics in a polymer near $T_{g}$, estimated by dielectric noise and single-molecule diffusion studies as being $>40 \mathrm{~nm}$ [18] and $\sim 100 \mathrm{~nm}$ [19], respectively. These size scales are much larger than that of an average cooperatively rearranging region (CRR) (where cooperative segmental motion occurs by collective motion of many polymer segments [20]) in neat polymers near $T_{g}$, $\sim 3 \mathrm{~nm}$ for PS and poly(vinyl acetate) [21,22]. Adding a small-molecule diluent to the polymer alleviates the requirements of cooperativity [16,17], reducing the size scale of an average CRR and, by implication, the length scale needed to encompass the breadth of the cooperative segmental dynamics and over which confinement effects are observed. This is consistent with Figs. 2 and 3 showing that moderate diluent levels (resulting in moderate reductions in the size scale of a CRR) reduce nanoconfinement effects while higher diluent levels (resulting in larger reductions in the size of a CRR) eliminate these effects in PS films down to thicknesses of $13 \mathrm{~nm}$.

Reduction of the bulk $T_{g}$ by diluents is not sufficient for reducing or circumventing nanoconfinement effects; independent of modifying $T_{g}$, diluents must reduce the cooperativity associated with segmental mobility, i.e., result in a "less fragile" [16,17] glass former. This point is demonstrated by reducing the $T_{g}$ of bulk PS $\left(M_{n}=\right.$ $263 \mathrm{~kg} / \mathrm{mol}$ ) by $20 \mathrm{~K}$ via addition of oligomeric PS ( $M_{n}$ of 0.8 or $1.3 \mathrm{~kg} / \mathrm{mol}$ ), yielding a PS blend with $M_{n}=$ $5.0 \mathrm{~kg} / \mathrm{mol}$. For example, a 38-nm-thick PS film with $M_{n}=5.0 \mathrm{~kg} / \mathrm{mol}$ (blend of PS from $M=263 \mathrm{~kg} / \mathrm{mol}$ and $M_{n}=1.3 \mathrm{~kg} / \mathrm{mol}$ samples, doped with $0.2 \mathrm{wt} \%$ pyrene) exhibits a $T_{g}$ that is reduced by $7 \mathrm{~K}$ compared to the bulk $T_{g}$ of $353 \mathrm{~K}$. This $7 \mathrm{~K}$ reduction is within error of the $5 \mathrm{~K}$ reduction expected for a 38-nm-thick unplasticized PS film (see Fig. 2). In addition, the strength of the transition at $T_{g}$ in this $5.0 \mathrm{~kg} / \mathrm{mol}$ PS is reduced with decreasing film thickness in a manner similar to that shown in Fig. 1(a). The results obtained in this study for the $5.0 \mathrm{~kg} / \mathrm{mol}$ PS system are consistent with those of Tsui and Zhang [23] who studied PS films of $M_{w}=13.7$ and $550 \mathrm{~kg} / \mathrm{mol}$ and mixtures thereof and found that the nanoconfinement effect is independent of molecular weight for supported PS films. [A very different system, poly(methyl methacrylate) on $\mathrm{SiO}_{x}$ substrates treated with hexamethyl disilazane, has reportedly shown [24] suppression of $T_{g}$-nanoconfinement effects for molecular weights of 5 and $12 \mathrm{~kg} / \mathrm{mol}$; further study of the different effects of molecular weight in this system is warranted.]

Small-molecule diluents also reduce nanoconfinement effects for P2VP on glass, with its attractive polymersubstrate interactions. Figure 4 shows the $T$ dependence
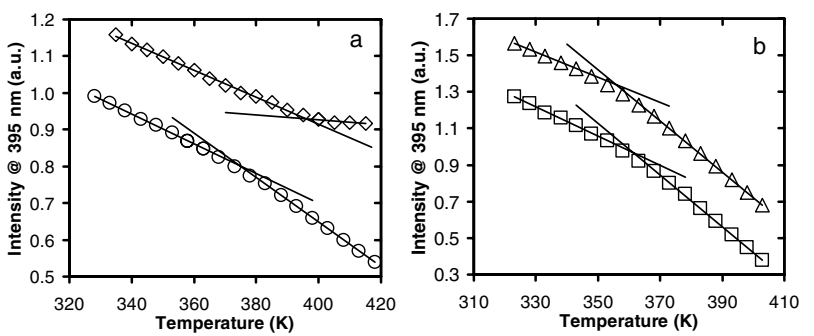

FIG. 4. Temperature dependence of the fluorescence intensity for P2VP films doped with pyrene. P2VP films doped with $0.2 \mathrm{wt} \%$ pyrene (a) are $1030 \mathrm{~nm}(\bigcirc)$ and $28 \mathrm{~nm}(\diamond)$ thick; P2VP films doped with $9 \mathrm{wt} \%$ pyrene (b) are $955 \mathrm{~nm}(\triangle)$ and $27 \mathrm{~nm}(\square)$ thick. Intensities have been normalized to $373 \mathrm{~K}$ and arbitrarily shifted vertically for clarity. 
of fluorescence for P2VP films doped with 0.2 and $9 \mathrm{wt} \%$ pyrene [25]. The bulk $T_{g} \mathrm{~s}$ as measured by fluorescence for 0.2 and 9 wt \% pyrene-doped P2VP films are 374 and $356 \mathrm{~K}$, respectively, in reasonable agreement with bulk $T_{g} \mathrm{~s}$ obtained by DSC of 372 and $355 \mathrm{~K}$, respectively. The $0.2 \mathrm{wt} \%$ pyrene-doped P2VP films exhibit a $22 \mathrm{~K}$ increase in $T_{g}$ upon reducing thickness from 1030 to $28 \mathrm{~nm}$ and a difference in the $T$ dependence of fluorescence above $T_{g}$. In contrast, there is a smaller increase in the $T_{g}$ of 9 wt \% pyrene-doped P2VP films, by $6 \mathrm{~K}$, upon reducing thickness from 955 to $27 \mathrm{~nm}$, with little difference in the strength of the transition at $T_{g}$. The increase in $T_{g}$ by $6 \mathrm{~K}$ also contrasts with the results of the $9 \mathrm{wt} \%$ pyrene-doped PS films which exhibit a thicknessindependent $T_{g}$ down to $13 \mathrm{~nm}$, indicating that while small-molecule diluents in a P2VP film on glass reduce the nanoconfinement effect, they do so to an extent different from PS films on glass. This is likely due to the greater cooperativity requirements at the $\mathrm{P} 2 \mathrm{VP}$-glass interface relative to PS films on glass, resulting in an increase in the size scale of an average CRR and an increase in the length scale over which nanoconfinement effects are observed in supported P2VP.

Finally, the thickness-independent $T_{g}$ for PS films with $9 \mathrm{wt} \%$ pyrene is consistent with Ref. [26] which reported a thickness-independent $T_{g}$ for PS films doped with $0.1 \mathrm{wt}$ fraction bispyrenyl propane (BPP). Although a high level of BPP should reduce bulk $T_{g}$, a $T_{g}$ of $373 \mathrm{~K}$ was reported by fluorescence, that of bulk, neat PS. (By not acknowledging that high dopant levels plasticize PS, Ref. [26] indicated no nanoconfinement effect on PS $T_{g}$.) With 0.2 wt $\%$ BPP in PS, we find a break in the $T$ dependence of fluorescence at $\sim 383 \mathrm{~K}, 10 \mathrm{~K}$ above the $T_{g}$ of neat PS. Reference [26] may have reported a thickness-independent $T_{g}$ of $373 \mathrm{~K}$ due to unrecognized effects: BPP yields an anomalous break in the $T$ dependence of fluorescence, leading to a high estimation of $T_{g}$; high BPP levels plasticize PS, reducing the $T_{g}$ inferred by fluorescence; and high BPP levels reduce the size scale over which nanoconfinement effects are observed. Reference [7] describes cases in which fluorescence yields anomalous $T_{g} \mathrm{~s}$, e.g., fluorescence from a label made from 1-pyrenylbutyl methacrylate yields correct $T_{g} \mathrm{~s}$ while that made from 1-pyrenylmethyl methacrylate does not. Thus, while a pyrene dopant is well suited for $T_{g}$ determination by fluorescence, BPP may not be [27].

*To whom correspondence should be addressed. Electronic address: j-torkelson@ northwestern.edu

[1] J. L. Keddie, R. A. L. Jones, and R. A. Cory, Europhys. Lett. 27, 59 (1994).

[2] S. Kawana and R. A. L. Jones, Phys. Rev. E 63, 021501 (2001).
[3] J. H. van Zanten, W. E. Wallace, and W. L. Wu, Phys. Rev. E 53, R2053 (1996).

[4] K. Fukao and Y. Miyamoto, Phys. Rev. E 61, 1743 (2000).

[5] G. B. DeMaggio et al., Phys. Rev. Lett. 78, 1524 (1997).

[6] C. J. Ellison, S. D. Kim, D. B. Hall, and J. M. Torkelson, Eur. Phys. J. E 8, 155 (2002).

[7] C. J. Ellison and J. M. Torkelson, J. Polym. Sci., Pt. B: Polym. Phys. 40, 2745 (2002).

[8] C. J. Ellison and J. M. Torkelson, Nat. Mater. 2, 695 (2003).

[9] J. A. Forrest, K. Dalnoki-Veress, J. R. Stevens, and J. R. Dutcher, Phys. Rev. Lett. 77, 2002 (1996).

[10] D. B. Hall, J.C. Hooker, and J. M. Torkelson, Macromolecules 30, 667 (1997).

[11] J. A. Forrest and K. Dalnoki-Veress, Adv. Colloid Interface Sci. 94, 167 (2001).

[12] K. Binder, J. Baschnagel, and W. Paul, Prog. Polym. Sci. 28, 115 (2003).

[13] J. A. Torres, P. F. Nealey, and J. J. de Pablo, Phys. Rev. Lett. 85, 3221 (2000).

[14] G. M. Wallraff and W. D. Hinsberg, Chem. Rev. 99, 1801 (1999).

[15] D. S. Fryer, S. Bollepali, J. J. de Pablo, and P. F. Nealey, J. Vac. Sci. Technol. B 17, 3351 (1999).

[16] R. Casalini et al., J. Polym. Sci., Pt. B: Polym. Phys. 38, 1841 (2000).

[17] K. Hamilton, Ph.D. thesis, Northwestern University, 1996.

[18] E.V. Russell and N. E. Israeloff, Nature (London) 408, 695 (2000).

[19] J. C. Quirin, A. C. Bartko, R. M. Dickson, and J. M. Torkelson, Polym. Prepr. (Am. Chem. Soc., Div. Polym. Chem.) 42, No. 2, 174 (2001).

[20] G. Adam and J. H. Gibbs, J. Chem. Phys. 43, 139 (1965).

[21] E. Hempel et al., J. Phys. Chem. B 104, 2460 (2000).

[22] U. Tracht et al., Phys. Rev. Lett. 81, 2727 (1998).

[23] O. K. C. Tsui and H. F. Zhang, Macromolecules 34, 9139 (2001).

[24] G. Kleideiter et al., Macromol. Symp. 145, 95 (1999).

[25] The greater temperature dependence of the fluorescence intensity in the glassy state compared with that of the rubbery state for the 28-nm-thick P2VP film in Fig. 4(a) has been reported for other P2VP and poly(isobutyl methacrylate) films of similar thickness [6]. Studies are under way to characterize the contributing factors of the unusual temperature dependence of the rubbery-state fluorescence observed in a nanoconfined polymer with attractive substrate interactions.

[26] C. C. White, K. B. Migler, and W. L. Wu, Polym. Eng. Sci. 41, 1497 (2001).

[27] Reference [26] infers $T_{g}$ from the $T$ dependence of a BPP excimer to monomer fluorescence intensity, $I_{E} / I_{M}$, describing the excimer as intramolecular. We find no excimer in $0.2 \mathrm{wt} \%$ BPP doped PS, ruling out an intramolecular excimer, nor in our $0.2 \mathrm{wt} \%$ pyrene-doped or pyrene-labeled systems. When we monitor the $T$ dependences of monomer intensity and $I_{E} / I_{M}$, identical $T_{g} \mathrm{~s}$ are obtained in higher pyrene content systems that exhibit mostly monomer but some intermolecular excimer fluorescence. 